\title{
La política interregional de la Unión Europea en el encuentro Asia-Europa (ASEM)*
}

\author{
Manuel de Jesús Rocha Pino**
}

\begin{abstract}
Resumen. En 1996 fue creado el Encuentro Asia-Europa (ASEM) con el fin de constituir un mecanismo de diálogo interregional inexistente hasta ese momento y que uniera a los dos extremos de la masa territorial euroasiática: Asia del Este (representada por el grupo ANSEA 3) y Europa (representada por la Unión Europea). En el momento de su creación fueron muchas las expectativas, pero con los años ha evidenciado un conjunto de limitantes que lo han vuelto inefectivo, al menos en el campo del diálogo político. En este trabajo se describe la particular experiencia de la diplomacia de la Unión Europea en el mecanismo del ASEM, el tipo de política interregional que ésta ha implementado y las contradicciones que ha tenido que tolerar frente a sus propias exigencias legales e institucionales. En el trabajo se argumenta que, no obstante sus limitantes, el ASEM puede ser un caso referencial sobre la reacción de la Unión Europea frente a la transformación que en términos históricos representa la emergencia política y económica de Asia en el sistema internacional.

Palabras clave: interregionalismo, ASEM, triada interregional, Comisión Europea, constructivismo
\end{abstract}

Abstract. During 1996 the Asia-Europe Meeting (ASEM) was created with the purpose of constituting a mechanism of nonexistent interregional dialogue until that moment and that united the both extremes of the eurasian territorial mass: East Asia (represented by group ANSEA 3) and Europe (represented by the European Union). The expectations erected at the moment of ASEM's creation were many, but with the years it has demonstrated a set of limits that has diminished its effectiveness, at least in the area of the political dialogue. In this paper is described the particular experience of the diplomacy of the European Union in the mechanism of the ASEM, the kind of interregional policy that this one has implemented and the contradictions that it has had to tolerate with respect to his own legal and institutional exigencies. In the paper it is argued that, despite its limits, the ASEM can be a referential case on the reaction of the European Union forehead to the transformation that in historical terms means the political and economic emergency of Asia in the international system.

Key words: interregionalism, ASEM, interregional triad, European Comission, constructivism

* El autor agradece a la doctora Esther Zapater Duque de la Universidad Autónoma de Barcelona los comentarios realizados a la presente investigación. Este trabajo se realizó gracias a una beca de estudios de posgrado otorgada por el Consejo Nacional de Ciencia y Tecnología de México (Conacyt).

** Universidad Autónoma de Barcelona, Institut Universitari d'Estudis Europeus. Correo electrónico: rochapino@yahoo.com 


\section{Introducción}

La Unión Europea utiliza diferentes estrategias para relacionarse con el mundo exterior. Estas herramientas abarcan desde diálogos estratégicos bilaterales, políticas de ampliación y de buena vecindad y un conjunto de diálogos interregionales, mecanismos todos ellos que ayudan a la instrumentación de la agenda global de la Unión. En este trabajo se describirá la importancia que tienen los diálogos interregionales o el interregionalismo en la instrumentación de dicha agenda global. Dada su naturaleza, la UE debe ser una promotora de dichos mecanismos. Sin embargo, existen diferencias cualitativas entre el interregionalismo que la Unión asume con ciertas regiones como América del Norte y Asia del Este (de creciente importancia económica y política) respecto a otras regiones más bien periféricas, como los llamados países ACP (África, Caribe y Pacífico) e incluso América del Sur.

En la investigación se utilizará un marco teórico constructivista para interpretar la trayectoria que ha mantenido el diálogo político en el caso específico del Encuentro Asia Europa (Asia Europe Meeting, organización mejor conocida como ASEM, por sus siglas en inglés) desde sus orígenes, y en la que se han presentado algunas inconsistencias y dificultades institucionales y legales para la propia acción exterior de la UE; tales tensiones han limitado el desarrollo de un diálogo político efectivo en el proceso del ASEM, orientando al aparato institucional de la UE a mostrar cierta tolerancia ante las inconsistencias surgidas con el fin de continuar con el proceso de diálogo interregional. En la investigación se argumenta que tales dificultades forman parte de un proceso histórico más amplio: la emergencia de un nuevo centro de poder mundial (Asia del Este) y la reacción que la UE ha mantenido ante tal proceso durante parte de la Posguerra Fría. ${ }^{1}$

\footnotetext{
${ }^{1}$ Hasta la cumbre ASEM 6 realizada en Helsinki en 2006, el Encuentro Asia Europa era conformado por 38 países, además de la Comisión Europea en tanto representante de la UE. Por la parte europea se encontraban los 25 miembros de la UE: Alemania, Austria, Bélgica, Chipre, Dinamarca, Eslovaquia, Eslovenia, España, Estonia, Francia, Finlandia, Grecia, Hungría, Irlanda, Italia, Letonia, Lituania, Luxemburgo, Malta, Países Bajos, Polonia, Portugal, Reino Unido, República Checa
} 


\section{El interregionalismo durante la Posguerra Fría}

\section{Los acuerdos interregionales}

El sistema bipolar durante la Guerra Fría inhibió el desarrollo de las relaciones interregionales más allá de las alianzas de seguridad transregionales y algunos diálogos establecidos por la Comunidad Económica Europea (CEE) con otros grupos regionales; pero tras la desaparición de la URSS, la proliferación de acuerdos regionales bajo un así denominado "nuevo regionalismo" incrementó el establecimiento de relaciones interregionales más allá de las relaciones grupo a grupo que tradicionalmente había sostenido la CEE. "A pesar que la UE es aún el principal actor en la creciente red de relaciones entre grupos regionales, el número de acuerdos interregionales más allá de las relaciones exteriores de la UE se ha incrementado" (Hänggi, 2000: 3). ${ }^{2} \mathrm{El}$ interregionalismo puede ser definido como el conjunto de "instituciones $\mathrm{u}$ organizaciones las cuales promueven el diálogo y la cooperación entre los países de diferentes regiones" (Chen, 2005: 364). Sin embargo, dado lo nueva que es esta área de estudio, la literatura sobre el tema sugiere una variedad de definiciones del término interregionalismo. Como propone Julia Schünemann, quizá lo más adecuado sea hablar de interregionalismos antes que del interregionalismo como un proceso unidireccional, "ya que en la sociedad internacional existen tipos y formas muy diversas del fenómeno que además son muy

\footnotetext{
y Suecia. Por la parte asiática se encontraban los miembros del Grupo ANSEA 3: China, Corea del Sur, Japón, Camboya, Brunei, Filipinas, Indonesia, Laos, Myanmar (país conocido también como Birmania), la Federación de Malasia, Singapur, Tailandia y Vietnam.

${ }^{2}$ El interregionalismo es producto del denominado "nuevo regionalismo", un proceso de construcción de instituciones regionales efectuado tras lo que se considera como la primera generación de este tipo de acuerdos durante los años cincuenta y sesenta (de la que formó parte la entonces Comunidad Europea del Carbón y el Acero). Este nuevo regionalismo expresa que, debido al creciente número de temas políticos con implicaciones transfronterizas, las organizaciones multilaterales han debido abarcar parte de la soberanía y recursos de sus países miembros para desarrollar cierta capacidad de acción frente a los problemas globales (Rüland, 2006: 48).
} 
difíciles de categorizar" (Schünemann, 2006). Esta diversidad puede apreciarse en las instituciones interregionales existentes, que pueden ir desde instituciones formales hasta estructuras flexibles sostenidas en la diplomacia de cumbres o en el establecimiento de canales de comunicación y pautas de comportamiento (Schünemann, 2006).

Para Chen Zhimin, las relaciones interregionales pueden tomar tres formas: como una relación intergrupo cuando se integra por dos grupos regionales formales, como en el caso del diálogo UE-ANSEA (Asociación de Naciones del Sureste de Asia); como una relación birregional si varios países de la región (no necesariamente todos) construyen un mecanismo común, como en el caso del ASEM; y como una relación transregional en tanto que los países de dos o más regiones establezcan un vínculo a través de una comunidad transregional más amplia (como la OTAN y APEC) (Chen, 2005: 366). Por su parte, Heiner Hänggi identifica tres tipos ideales de acuerdos interregionales:

a) Relaciones entre grupos regionales. Los diálogos grupo a grupo, como el sostenido entre la UE y la Asociación de Naciones del Sureste de Asia (ANSEA) desde los años setenta y ochenta, son el prototipo de este tipo de acuerdos, creando un modelo para que otras regiones establezcan sus propios acuerdos interregionales grupo a grupo. A la vez, la CEE usó dichas relaciones interregionales para promover las relaciones intrarregionales entre sus miembros. Con el fin de la Guerra Fría y el advenimiento de llamado "nuevo regionalismo", otras regiones como América Latina, Oceanía y el Sureste de Asia procedieron a establecer relaciones entre ellos.

b) Acuerdos birregionales y transregionales. Las relaciones interregionales y transregionales de este tipo emergen en el contexto de triángulo interregional o "triada". El concepto de triada tiene sus raíces en las relaciones trilaterales establecidas entre los Estados Unidos, la CEE y Japón durante los años setenta y trata de reflejar la emergencia de un orden económico mundial dividido en un triángulo global: América del Norte, Europa Occidental y Asia del Este. Entre las razones para el surgimiento del triángu- 
lo interregional pueden citarse el fin de la Guerra Fría, la aparición del nuevo regionalismo y la emergencia de Asia del Este como el tercer centro en importancia de la economía mundial. Entre los principales diálogos interregionales pueden citarse: la APEC (surgida en 1989), el ASEM, las Cumbres América LatinaUnión Europea iniciadas en 1999, la Cumbre del Cairo ÁfricaEuropa iniciada en el 2000 y el Foro Asia-América Latina (EALAF) creado oficialmente en el año 2000.

c) Hibridos entre el interregionalismo y el bilateralismo. Estas relaciones interregionales se establecen cuando el esquema del diálogo involucra tanto a los socios regionales (las organizaciones) como a los países miembros del grupo en su carácter individual. La participación de los miembros de la UE en el Encuentro Asia Europa hace que el ASEM sea un caso híbrido, conjugando tanto bilateralismo como una relación interregional. Como veremos, en él están representados tanto los países de forma individual, como la UE en tanto organización (en la cumbre ASEM 6 se admitió a la Secretaría General de la ANSEA como nuevo miembro) (Hänggi, 2000: 3-11).

\section{La teoría internacional y el interregionalismo}

Una teoría sobre el interregionalismo aún resulta incompleta. Los estudios teóricos existentes sobre el tema en realidad reflejan el discurso teórico (o metateórico) reciente en las relaciones internacionales, que incluye desde el discurso neorrealista y el liberalinstitucionalismo hasta el constructivismo, y en ocasiones enlaza elementos de dichos enfoques con el fin de formular nuevas aproximaciones de análisis sobre un conjunto de fenómenos que emergen en el sistema internacional (con el riesgo de perder el rigor teórico necesario que ello conlleva): "las variaciones sobre dichas mezclas dependen del contexto así como de los factores cognitivos, como por ejemplo las interacciones previas, las experiencias históricas y las expectativas resultantes en el papel a desempeñar como resultado en las percepciones del actor" (Hänggi, Roloff y Rüland, 2005: 10). 
a) Realismo. Para los realistas el ASEM es resultado de la redistribución de poder y de los cálculos al respecto por parte de los actores clave que impulsaron el proyecto. Desde la perspectiva realista, éste es sólo un foro diplomático intergubernamental en el que los Estados nacionales continúan siendo los principales actores y sus intereses la principal motivación para el mantenimiento del diálogo y los planes de cooperación. Sin embargo, el ASEM no ha despertado muchas preocupaciones en los Estados Unidos, tanto por la diversidad de opiniones de sus miembros como por la carencia de objetivos político-militares bien definidos en el foro (Brobow, 1999: 103-128).

b) Liberalinstitucionalismo. Para esta corriente teórica, los Estados crean a las organizaciones internacionales para solucionar sus problemas a través de los mecanismos informales o de regímenes cooperativos ("reduciendo costos de transacción y solucionando los problemas de distribución") (Song, 2007: 69). Para el liberalinstitucionalismo el ASEM forma parte de un regionalismo ascendente que usa a los foros interregionales para enfrentar los retos de la globalización.

c) Constructivismo. El constructivismo estudia la construcción de una identidad a partir de los papeles que asumen los actores internacionales en un proceso de socialización dado. En términos de Gilson, "El interregionalismo representa la interacción de una región con otra". Aunque el ASEM no es la reunión de dos regiones preexistentes (Asia y Europa), ${ }^{3}$ la decisión de articular el concepto de asociación entre iguales representó un intento explícito por parte de los Estados miembros por no seguir un camino transregionalista como el modelo de la APEC, sino crear un diálogo de región a región. El interregionalismo es frecuentemente caracterizado como un "doble proyecto regional" que responde a la necesidad de reunir un porcentaje siempre mayor de recursos en el reconocimiento de otra dinámica interregional o global (Gilson, 2005: 309). Desde una perspectiva constructivista, tanto "Europa" como "Asia" en el ASEM son dos conceptos imaginados que, al establecer una relación, comienzan a elaborar una identidad propia frente al "otro". El liberalinstitucionalismo está de acuerdo con esta perspectiva, pero afirma que dicho proceso de construcción de identi-

\footnotetext{
${ }^{3}$ Cursivas del autor.
} 
dades tiene lugar después de consolidado el proceso de cooperación interregional, mientras que el constructivismo establece que la existencia de intereses compartidos debe ser antecedida por la construcción de una identidad (Song, 2007: 69). “La cooperación interregional es un fenómeno mediático: las regiones constantemente tienen que reinterpretar sus respectivas acciones y renegociar la realidad en la cual operan" (Schünmann, 2006).

La diplomacia de cumbres puede ser un medio aparentemente efectivo en este proceso de construcción de identidades a través de la socialización, aunque en ocasiones tales actos se vean restringidos a la participación de las élites políticas de los participantes, marginando del proceso político (no mediático) a parte de la opinión pública de ambas regiones.

\section{El triángulo interregional o "triada"}

La literatura sobre el interregionalismo se ha centrado fundamentalmente en el análisis del triángulo interregional o "triada", atribuyendo cinco funciones esenciales a los foros interregionales a partir de este análisis: como balance frente a actores; como partícipes en la construcción de instituciones; como racionalizadores y generadores de una agenda internacional; y como constructores de identidades. Estas cinco funciones pueden converger con las tres propuestas teóricas mencionadas (Rüland, 2006: 48). ${ }^{4}$ El triángulo interregional es conformado actualmente por la OTAN, el Foro Económico Asia Pacífico (APEC) y el ASEM.

\footnotetext{
${ }^{4}$ De acuerdo a Jürgen Rüland, la función de balance es diferente a la noción realista del término pues responde a una estrategia diseñada para ajustar el desequilibrio político y económico en el interior de la triada reajustando la influencia institucional. En este sentido, “APEC es la respuesta de la Cuenca del Pacífico a lo que parece una emergencia del poder europeo; ASEM es una respuesta europea a la APEC; y tanto la Nueva Agenda Transatlántica como el TLCAN son la respuesta estadounidense a ASEM". La construcción de instituciones se refiere al establecimiento de nuevos mecanismos de interacción internacional al tiempo que estimula la cooperación intraregional. Como racionalizador se le atribuyen al interregionalismo funciones subsidiarias respecto a las organizaciones multilaterales, tanto aportando nuevos temas a la agenda como colaborando para alcanzar acuerdos. La construcción de identidades se refiere a los procesos cognitivos en el sentido constructivista (Rüland, 2006: 48-49).
} 
De los tres organismos, la OTAN es el único formado en el contexto de la Guerra Fría, aunque también es el que ha desarrollado el más alto grado de institucionalización. ${ }^{5}$ Conforme avanzaban las décadas de los ochenta y los noventa, las relaciones entre el triángulo económico mundial se orientaron a una competencia mutua entre ellos en lugar de que se establecieran vínculos plenos de cooperación. ${ }^{6}$ Los Estados Unidos fueron los primeros en aproximarse a una de las partes, en el caso del Pacífico, y de ahí nació el foro económico de la APEC en 1994 con el fin de establecer un área de libre comercio bajo la noción estadounidense de una región Asia-Pacífico (entendida como un espacio vital en el proceso de expansión estadounidense, integrando de manera un tanto arbitraria a Asia con los países latinoamericanos miembros del foro). En lo que respecta a la agenda transatlántica, Estados Unidos y la UE establecieron un nuevo esquema de relaciones bajo la Nueva Agenda Transatlántica en 1995.

Hacia 1996 quedaba claro que el vértice más débil del triángulo interregional era el euroasiático, y fue entonces que se propuso la creación del ASEM. De los tres acuerdos interregionales que conforman la "triada", el euroasiático es el único que se propone como una asociación de relaciones simétricas entre sus participantes, puesto que tanto en el caso de la APEC como en el de la Nueva Agenda Transatlántica, es una sola potencia, la estadounidense, la que mantiene una clara hegemonía sobre la región "socia". A su vez, mientras que la OTAN era un producto de la Guerra Fría, los otros dos foros interregionales aparecieron después de ésta.

\footnotetext{
${ }^{5}$ Políticamente la OTAN es una institución intergubernamental, por lo que su sistema de toma de decisiones se basa en el consenso por unanimidad manteniéndose la soberanía de sus miembros; en cambio, la APEC es un foro internacional en el que las economías de los países que la componen cooperan en comercio y liberalización de inversiones. La APEC no es una institución internacional en términos legales ya que no se fundamenta en algún tratado o acuerdo internacional ni posee un mecanismo de toma de decisiones propio (Chen, 2005: 366-367).

${ }^{6}$ La estructura de la economía global indica que la globalización no es realmente global, dada la concentración de la actividad económica en América del Norte, la UE y Asia del Este, áreas que en conjunto concentran las tres cuartas partes del comercio global y generan el $90 \%$ de los flujos de inversión extranjera directa. El establecimiento de un "triángulo interregional" de este tipo también refleja una creciente marginalización de las economías más débiles.
} 
Desde una perspectiva realista, articulada sobre todo por la UE (la parte asiática se interesaba fundamentalmente en ampliar sus relaciones económicas con Europa), el ASEM podría servir para proteger los intereses europeos frente a la hegemonía estadounidense en el Pacífico (Dent, 2004: 215).

Aunque los foros interregionales aumentaron durante la década de los noventa, sería erróneo asumir que esto refleja una trayectoria lineal en la institucionalización de las relaciones internacionales. Los países en desarrollo del Sureste de Asia prefirieron mantener una baja institucionalización en sus relaciones interregionales, optando por un esquema de foro consultivo de manera similar a las organizaciones multilaterales a las que pertenecían (como la ANSEA), y rechazando decisiones que involucraran demasiados compromisos, así como los altos costos económicos que comúnmente se asocian con el elaborado paquete organizacional de un proceso formal (Hänggi, Roloff y Rüland, 2005: 8).

\section{Aproximaciones simétricas y asimétricas \\ al interregionalismo por parte de la UE}

En contraste con sus primeros años de existencia, en que las preocupaciones de las Comunidades Europeas se centraban en tomar posiciones comunes sobre temas comerciales y sus relaciones exteriores se orientaban hacia sus antiguas colonias, en la actualidad la Unión Europea ha tenido que tomar un papel activo como actor global en el sistema internacional, asumiendo una agenda que involucra tanto cuestiones económicas (liberalización del comercio, promoción de la integración regional, cooperación para desarrollo) como políticas (democracia y gobernanza global, derechos humanos, medio ambiente). En los últimos años, el tema de la seguridad global y las políticas concernientes a ella se ha agregado a dicha agenda, por lo que la UE se ha visto obligada a participar tanto como le sea posible en el entorno internacional. Esa capacidad de actuación (actorness) le puede garantizar su propia seguridad interna y el de su periferia; por lo anterior la presencia de la UE en el entorno internacional se ha convertido en una necesidad, en tanto que su ausencia en algún espacio o región podría 
interpretarse como una expresión de debilidad o un vacío de poder por parte de la Unión.

Existen básicamente cuatro mecanismos mediante los cuales se instrumenta la acción exterior de la Unión Europea: por medio de ampliaciones, agregando nuevos miembros a la Comunidad con el fin de crear un entorno europeo estable; por medio de políticas de vecindad que buscan dar estabilidad a su periferia (en los casos del llamado espacio Euromediterráneo y de los diálogos con Ucrania o Bielorrusia); mediante acuerdos bilaterales con grandes potencias o economías emergentes (como la Nueva Agenda Transatlántica o los diálogos o asociaciones estratégicas con Rusia, China y Japón, o los acuerdos comerciales establecidos con México y Chile), y mediante el interregionalismo, estableciendo acuerdos con otras organizaciones regionales. Como veremos, en los últimos diez años la política interregional de la UE se ha convertido en un componente relevante de su política exterior, siendo la Comisión Europea la instancia encargada de negociar los acuerdos interregionales y de vigilar su cumplimiento en coherencia con los principios políticos y jurídicos de la Unión (Hettne y Söderbaum, 2005: 535). De la misma manera, el interregionalismo también podría contribuir para coordinar la acción colectiva de los miembros de la Unión en el esquema de la Política Exterior y de Seguridad Común (PESC), salvaguardando valores comunes (como los criterios de gobernanza global de la UE) y la independencia e integridad de la Unión en conformidad con los principios de la Carta de la ONU, al tiempo que se fortalece su seguridad (Reiterer, 2006: 352).

Para Björn Hettne y Frederik Söderbaum, la política exterior europea se puede calificar como blanda (basada en instrumentos económicos, de diálogo y diplomáticos) en contraste con otro conjunto de políticas duras (como la coerción o las represalias militares), y puede ser formulada a partir dos tipos ideales: asociándosele, por un lado, con el idealismo (normativismo) de los valores políticos que la UE trata de difundir, y por el otro con las políticas basadas en los intereses nacionales de sus miembros (la realidad tras la retórica política). Las dos orientaciones anteriores generan diferentes maneras de instrumentar dicho poder blando. Entonces, a partir del concepto de poder blando es necesario hacer una distinción entre lo que puede de- 
nominarse "poder civil" (civilian power) e "imperalismo blando" (soft imperalism). Ambos tipos ideales definidos por Hettne y Söderbaum son llevados a cabo en los cuatro mecanismos de la acción exterior europea (Hettne y Söderbaum, 2005: 536).

La utilización del poder civil en la política exterior europea se debe a que el propio modelo de construcción intrarregional que ha llevado a cabo la UE se ha extendido a su acción exterior, y el sistema internacional en que busca actuar la Unión sería uno modelado bajo una normatividad similar. "La diferencia entre el poder civil y el imperialismo blando yace en la importancia total de valores y normas en las negociaciones que son llevadas a cabo de manera simétrica, mediante diálogos o, en su defecto, mediante la imposición" (Hettne y Söderbaum, 2005: 538). Al ejercer su capacidad de acción, la UE debe influir en el sistema internacional a través de una agenda que corresponda con su propio modelo de integración regional basado en los derechos humanos, la buena gobernanza, el respeto por los intereses de los diversos actores del grupo interregional, la igualdad, el multilateralismo y el derecho internacional. El sistema institucional de la UE se encuentra obligado a ello, tanto por los valores políticos que contiene como por una obligación jurídica: en el Libro Blanco $\mathrm{La}$ Gobernanza Europea, redactado a instancias de la Comisión Europea en el año 2000, se establece que
El vínculo entre la gobernanza europea y la gobernanza mun- dial debería permitir hablar a la Unión más a menudo con una sola voz. La fijación de prioridades para el examen de denuncias sobre infracciones del Derecho comunitario optimizará el impacto de la labor llevada a cabo por la Comi- sión como guardiana del Tratado (en referencia al Tratado de la Unión Europea) (Comisión Europea, 2001: 35).

Dicho ordenamiento jurídico determina la presencia de la UE en el sistema internacional. ${ }^{7}$

${ }^{7}$ En 1995, la Comisión definió las medidas que pueden ser tomadas en respuesta a violaciones graves en derechos humanos o interrupciones serias de un proceso democrático: 
Al elegir una relación simétrica con base en la igualdad con sus socios asiáticos en el ASEM, la UE ha elegido el poder civil como el principal esquema orientador de sus políticas. Pero la UE también es capaz de ejercer mecanismos de presión enérgicos en espacios interregionales francamente asimétricos, como en el caso de su relación con los países de África, el Caribe y el Pacífico (ex colonias europeas conocidas como países ACP) o con América Latina. En estos casos la política aplicada puede definirse como imperialismo blando:

[...] por imperialismo blando nos referimos al poder blando aplicado de manera dura, esto es, en una forma asimétrica de diálogo o incluso la imposición o uso estratégico de normas y condicionalidades llevadas a cabo por razones de su autointerés, en lugar de la creación de un genuino diálogo interregional (sostenible de una real gobernanza global) (Hettne y Söderbaum, 2005: 537).

La presencia de la UE en diferentes ámbitos del sistema internacional refleja su capacidad de actuación. Dicha capacidad conduce su comportamiento externo, debe tener un gran alcance de acción y espacio de maniobra y, en algunos casos, incluso personalidad legal. Con base en su capacidad de actuación, la UE puede verificar si el proyecto de gobernanza europea tiene alguna influencia en el sistema internacional.

- Alteración de los contenidos de los programas de cooperación o de los canales usados.

- Reducción de los programas culturales, científicos o técnicos de cooperación.

- Posponer encuentros conjuntos con el Comité.

- Suspensión de contactos bilaterales de alto nivel.

- Posponer proyectos nuevos.

- Embargos comerciales.

- Suspensión de venta de armas y de cooperación militar.

- Suspensión de cualquier tipo de cooperación.

Sin embargo, la Comisión prefiere sanciones positivas, como entrar en un diálogo con el gobierno involucrado, en lugar de las sanciones negativas de suspensión o terminación de acuerdos (Reiterer, 2006: 234). 
C. Brethorn y J. Vogler identifican cinco requerimientos para la capacidad de actuación o actorness.

1. Misión compartida para un conjunto de valores compartidos y principios.

2. Habilidad para identificar prioridades políticas y para formular políticas coherentes.

3. Habilidad efectiva para negociar con otros actores en el sistema internacional.

4. Disponibilidad y capacidad para utilizar instrumentos políticos.

5. Legitimación doméstica del proceso de toma de decisiones y prioridades en relación con la política exterior (citados por Hettne y Söderbaum, 2005: 537).

La UE tiene una inclinación a formalizar e institucionalizar las relaciones entre los grupos regionales en los que participa en lugar de orientarse a los más difusos contactos bilaterales, pero por motivos pragmáticos o estratégicos sus formas de relación con los grupos interregionales varían, tendiendo con ello a instrumentar diferentes tipos de políticas, ya sean de poder civil o imperialismo blando, dependiendo de la naturaleza de sus socios. De esa forma, la UE usa los acuerdos interregionales para reducir la incertidumbre en el sistema internacional, proveer canales de comunicación y crear normas de comportamiento aceptable de acuerdo a sus valores. Sin embargo, la UE no puede ser interpretada como un sistema político monolítico ya que, en referencia a su política exterior, el poder sobre la toma de decisiones es compartido entre los Estados miembros y la Comunidad: las instituciones de la Unión hasta el momento han carecido de la autoridad y legitimidad suficientes para imponer acuerdos o instrumentar una política exterior diferente a los necesarios consensos establecidos entre los Estados miembros. La diversidad de intereses se expresa de manera notable en la relación de la UE con el Sureste de Asia, relación divida entre la agenda política de la Unión y los intereses específicos de cada uno de los miembros, que pueden ser ex países coloniales con intereses políticos y económicos como Reino Unido, Francia o Portugal; países preocupados por las relaciones económicas 
con Asia como Alemania, o con intereses más bien centrados en la protección de los derechos humanos como los escandinavos (Forster, 1999: 789).

Como veremos, el ASEM es un organismo en el que la UE aplica una voluntad de relación simétrica de manera más firme y en algunos casos obligada por las coyunturas políticas, e incluso con el riesgo de perder cierta coherencia en su política exterior al tener que tolerar la conducta de ciertos actores cuyos regímenes de hecho son autoritarios y, en teoría (y en la ley), no podrían relacionarse con la UE en ningún espacio de diálogo. Ello convierte al ASEM en una excepción en las relaciones interregionales establecidas por la Unión, ya que en la consolidación del vínculo asociativo con los países emergentes de Asia del Este, la Unión ha visto cuestionada la legitimidad de su agenda en la práctica (Forster, 1999a: 798). El principio de condicionalidad, presente en el Acuerdo de Cotonou que involucra a todos los países $\mathrm{ACP}$ (otro ejemplo es la "cláusula democrática" contenida en el acuerdo de libre comercio México-UE), no ha tenido el mismo efecto político en los países asiáticos y hasta la fecha no ha podido establecerse un acuerdo similar (Reiterer, 2006: 239). ${ }^{8}$

ASEM es frecuentemente señalado como un interregionalismo entre iguales. La visión de la UE es que la asociación Asia-UE debería ser vista como un lugar de encuentro en el que mucho puede ser ganado a partir de diálogo y el intercambio y [reconociendo] que no existe un solo "modelo europeo" de gobernanza social (Hettne y Söderbaum, 2005: 548).

En el caso del ASEM, el poder civil se usa de una manera menos asertiva, influyendo en los mecanismos de diálogo y consenso en lu-

\footnotetext{
${ }^{8}$ El Acuerdo de Cotonou (capital de Benin) es el tratado establecido entre la UE y los países ACP en el año 2000 (los ACP son un conjunto de 78 países que en 1975 establecieron la llamada Convención de Lomé, tratado que fue sustituido por el Acuerdo de Cotonou). El Acuerdo de Cotonou entró en vigor en 2002 y es el tratado de cooperación para el desarrollo más grande establecido entre la UE y las ex colonias ACP. Entre las diferencias con la Convención de Lomé, el nuevo acuerdo incluye temas comerciales como las uniones aduaneras y un mayor énfasis en el tema de la protección de los derechos humanos, la sociedad civil y la gobernanza en los países involucrados.
} 
gar de hacerlo directamente en la voluntad y el comportamiento de los socios asiáticos que son vistos como "iguales".

El ASEM es uno entre varios mecanismos disponibles para la política exterior de la UE. Dada la diversidad de sus socios asiáticos, la UE utiliza al foro interregional para manejar sus relaciones económicas y políticas con un conjunto heterogéneo de países emergentes. De la misma forma, no se pretende que dicha herramienta reemplace a otros acuerdos ya que desde el punto de vista institucional, el ASEM representa sólo un momento histórico en la construcción de esta variedad de mecanismos diplomáticos. Sin embargo, en la práctica real el organismo cuenta con un alcance limitado ya que parece existir un mayor interés en el lado europeo que en el asiático por desarrollar las potencialidades del ASEM, entre otras razones, dada la mayor capacidad y poder de la diplomacia europea (Dent, 2004: 225). A decir de Chistopher Dent: "mientras ASEM sirve para reforzar los órdenes multilaterales existentes, hasta la fecha ha tenido un impacto limitado en ayudar a desarrollarlos tanto en las áreas de la gobernanza existentes como en las nuevas" (Dent, 2004: 223). Para Sebastián Bersick, la cooperación interregional del proceso ASEM más bien promueve una "multiporalización cooperativa del sistema internacional y supone una alternativa multilateral para el orden de las relaciones internacionales en contraste con un modelo unipolar" (citado por Schünemann, 2006).

Lo anterior es algo que puede verificarse ante los consensos alcanzados en el proceso tras los atentados terroristas del 11 de septiembre de 2001 en los Estados Unidos: si bien el ASEM se ha orientado hacia la cooperación en materia de lucha contra el terrorismo, siguiendo la tendencia de los últimos años de incorporar cuestiones de seguridad contra las llamadas amenazas no convencionales en los diálogos políticos de alto nivel, al mismo tiempo el foro no se ha inclinado a adoptar o apoyar las mismas políticas de la lucha antiterrorista estadounidense. La multipolarización de las relaciones internacionales a través de un diálogo interregional como el ASEM no involucra un comportamiento de balance realista por necesidad. 


\section{ASEM como parte de la acción exterior de la Unión Europea}

\section{ASEM y las relaciones euroasiáticas}

La CEE inició encuentros bianuales de ministros de relaciones exteriores con la ANSEA en 1978, firmando un Acuerdo de Cooperación con la Asociación en 1980. El interés de la UE por Asia resurgió en los años noventa debido a las altas tasas de crecimiento que comenzaban a experimentar economías como las de Corea del Sur, Hong Kong, Singapur o Taiwán. Dicho interés se refleja en los documentos y comunicaciones de la Unión al respecto. El primero, aparecido en 1994, fue titulado "Una Nueva Estrategia para Asia" y cubría una variedad de temas de interés conjunto, desde el desarrollo sustentable, la protección del ambiente e investigaciones médicas, hasta la cooperación para la estabilidad de la economía global. Este acercamiento con Asia coincidió con la intensificación de las relaciones de la Unión con el exterior, en el que cambiaba su papel de una comunidad económica a uno como actor global. El Tratado de Maastricht de 1992 dotó a la UE de un esquema legal para el desarrollo de la Política Exterior y de Seguridad Común (PESC) y de las bases para una cooperación más profunda con terceros participantes, entre ellos los países asiáticos. La Nueva Estrategia para Asia fue un instrumento diseñado para mejorar la efectividad de las políticas instrumentadas por la EU a través de un canal interregional que serviría para suplementar los acuerdos bilaterales con los países de esa región y la participación en instituciones multilaterales. El argumento principal era la protección de los intereses de la EU en Asia, así como el bienestar económico interno de la Comunidad. ${ }^{9}$ Como afirman Richards y Kirkpatrick, estas preocupaciones generaron una doble "narrativa" que sirvió como punto de partida en las relaciones interregionales euroasiáticas: entre una Asia

\footnotetext{
${ }^{9}$ La existencia de una fuerte alianza transatlántica y la creación de la APEC en 1992 estimularon el interés europeo por contar con un mecanismo similar en sus relaciones con Asia, pero al contrario del carácter militar de la OTAN y económico de la APEC, el ASEM buscó extender sus intereses desde lo político hasta la seguridad, la economía y la cultura (Chen, 2005: 368).
} 
ascendente y la necesidad, más amplia, de un cuidadoso posicionamiento por parte de Europa en el mundo; ello constituyó un cálculo político que, desde entonces, "tuvo un profundo impacto, tanto en el análisis como en las prácticas políticas de las relaciones Asia-Europa" (Richards y Kirkpatrick, 1999: 701). ${ }^{10}$

El documento anterior fue sustituido hasta el año 2001 con el comunicado de la Comisión Europea titulado "Europe and Asia: A Strategic Framework for Enhnanced Partnership". En el comunicado se clarificaron los términos del intercambio interregional que ya sostenían ambos actores desde 1996 y se estableció que sus principales objetivos serían mantener la paz y la estabilidad en Asia, la cooperación económica y la inversión, la lucha contra la pobreza y la promoción de los derechos humanos y la democracia, así como mantener a la ANSEA como socio económico y político clave de la UE. El ASEM no sustituiría, sino reforzaría al diálogo oficial UE-ANSEA. Sin embargo, mientras que en el discurso la UE reconocía desde entonces a Asia como una de sus prioridades principales, en los años posteriores sus recursos se han enfocado hacia sus propias reformas internas, hacia los países recientemente incorporados a la UE mediante la ampliación y hacia el espacio mediterráneo. Hasta el momento, la UE nunca ha establecido una política común respecto a Asia ni ha nombrado a un enviado especial para la región (Oudjani, 2006: 337). ${ }^{11}$

${ }^{10}$ Como afirman Richards y Kirkpatrick, tales cálculos políticos se alcanzaron por consenso al interior de la comunidad: "La política asiática se construyó sobre los desarrollos internos asociados con la profundización en la integración europea, así como la creación de nuevos esquemas para la gestión externa de la economía política global que se derivaron de la acción colectiva de la Comisión, los mandatos de los Estados miembros y la colaboración con la comunidad de negocios" (Richards y Kirkpatrick, 1999: 701).

${ }^{11}$ Los objetivos y principios de cooperación de la UE pueden resumirse en seis puntos:

- Contribuir a la paz y la estabilidad de la región.

- Fortalecer el comercio mutuo y los flujos de inversión.

- Promover el desarrollo de las regiones menos prósperas.

- Contribuir a la protección de los derechos humanos y a la difusión de la democracia, la buena gobernanza y el estado de derecho.

- Construir asociaciones y alianzas globales.

- Fortalecer el conocimiento de Europa en Asia (Oudjani, 2006: 346). 
En 2004 se publicó un "Asia Strategic Paper" que serviría de esquema para las relaciones de la EU hacia la región durante los años 2005-2006; sin embargo, a pesar de los numerosos documentos y comunicados al respecto, la UE aún mantiene poco interés en ella. De acuerdo a Julie Gilson, esto puede deberse a varias causas: el tiempo y recursos ocupados en el propio proceso de integración de la UE, una carencia -a excepción de la protección de derechos humanos en Myanmar y China- de temas prioritarios en la región y la multiplicación de foros internacionales que tratan temas similares a los del ASEM (Gilson, 2005: 318). De la misma forma, la idea de que el ASEM fortalece al vértice euroasiático del triángulo interregional se ha convertido en una razón esencial para el mantenimiento del proceso de diálogo, "la tripolarización de la economía mundial se ha convertido en el mantra de los avances de ASEM" (Gilson, 2005: 313).

\section{Una breve historia del ASEM a través de sus cumbres}

El proceso de cooperación ASEM debe ser entendido como un diálogo informal y multidimensional divido en tres pilares: uno político, uno económico y uno cultural, con una orientación más reciente hacia la colaboración en la lucha contra el terrorismo (Alecu de Flers y Regelsberger, 2005: 332). La estructura de pilares es influencia directa de la existente en la UE.

Por lo general, se considera al primer ministro de Singapur Goh Chok Tong como el primer impulsor formal para la creación del proceso ASEM al sugerir el establecimiento de un diálogo interregional eurasiático durante una visita oficial a Francia en 1994, idea que fue retomada por Goh durante su discurso en el Foro Económico de Davos en 1995. Poco después, el Consejo Europeo dio una respuesta positiva a la propuesta como parte de la Nueva Estrategia para Asia. La primera reunión del proceso fue fechada para el año siguiente en Tailandia, algo que recibió el beneplácito del Parlamento Europeo (European Background Study, 2006: 30). La primera cumbre del ASEM, realizada en 1996 en Bangkok, fue saludada como un éxito por todos los participantes al ser la primera ocasión en que líderes europeos y asiáticos se reunían como iguales. La cumbre fue acompañada por una larga lista de actividades y proyectos 
de los cuales ha resaltado la creación de la Fundación Asia-Europa (ASEF) en Singapur en 1997, debido a la continuidad que ha mantenido hasta la fecha. La ASEF se conformó con el fin de facilitar la articulación de redes entre las organizaciones de la sociedad civil establecidas en las dos regiones. Sin embargo, el optimismo de la cumbre fue más emotivo que basado en hechos concretos, simbolizando el nuevo estatus que Asia comenzaría a disfrutar en el sistema internacional en tanto región emergente (European Background Study, 2006: 32).

La cumbre ASEM 2 se reunió en Londres en 1998, en el contexto de la crisis económico-financiera ocurrida en el Sureste de Asia el año anterior y cuyos efectos políticos y sociales seguían sintiéndose especialmente entre los miembros de la ANSEA. La cumbre tuvo como tema principal las consecuencias de la crisis. La UE se limitó a respaldar las medidas sugeridas por el Fondo Monetario Internacional a los afectados, pero sin intervenir realmente con algún programa de inversión a largo plazo para que los países más dañados pudieran reconstruir sus economías.

Por su parte, ASEM 3 se llevó a cabo en Seúl en octubre del año 2000. El tema de la cumbre nuevamente fue monopolizado por una coyuntura, en este caso la cuestión de las dos Coreas (el presidente surcoreano había ganado ese año el Premio Nóbel de la Paz), además de ciertos desacuerdos entre los países europeos sobre su aproximación a Corea del Norte y un nuevo rechazo europeo a la participación de Myanmar en el ASEM (Aggarwal, Vinod y Min Gyo Koo, 2005: 246).

La cumbre ASEM 4 se llevó a cabo en 2002 en Copenague y se enfocó a la lucha contra el terrorismo, la guerra de Irak y la proliferación nuclear en Corea del Norte, temas que monopolizaron la atención del diálogo.

Por otro lado, ASEM 5, realizada en Hanoi en 2004, sirvió como escenario de los desacuerdos por la incorporación de Myanmar al proceso, un tema que se había discutido desde la cumbre anterior. Mientras que la parte europea se negaba a aceptar la incorporación del régimen militar de Myanmar, dado que las propias leyes de la Unión prohíben el trato con regímenes autoritarios, la ANSEA condicionó la aceptación birmana a la incorporación de los nuevos miembros de la UE al Encuentro. Estos nuevos países eran producto de la ampliación de la UE 
del año 2004. Finalmente, la UE aceptó la incorporación de Myanmar en el ASEM con la condición de que este país asistiera a las reuniones con un nivel muy bajo de representación, un hecho que, si bien no afecta al funcionamiento de la política exterior de la UE, sí le resta algo de la coherencia que persigue. Es claro que para la UE resultó más importante mantener el proceso que prestar demasiada atención a un país marginal como Myanmar, pero también es obvio que la presión por parte de la ANSEA influyó en la negociación final.

La cumbre ASEM 6, llevada a cabo en octubre de 2006, no tuvo la orientación un tanto monotemática de las reuniones anteriores, sino que se centró en tres aspectos: el establecimiento de una agenda de temas de carácter global en los cuales centrar la cooperación (en donde resalta la colaboración en materia medioambiental, específicamente en el tema del cambio climático); la reforma de los métodos de trabajo del proceso; y la segunda ampliación del ASEM (Abad Quintanal, 2007: 139). ${ }^{12}$ Durante la cumbre se acordó la creación de una Secretaría Virtual del ASEM para agilizar el intercambio de información entre los miembros y dar seguimiento a los programas de cooperación en los periodos entre las cumbres bianuales. La creación de dicha Secretaría Virtual evidencia una flexibilización por parte de los países que se oponían a la institucionalización del ASEM y resulta "una indicación de la erosión de la naturaleza informal del proceso de cooperación Asia-Europa", algo necesario si consideramos a los nuevos actores incluidos en la nueva ampliación del foro (Loewen, 2007: 29). En la ampliación del ASEM de 2006 fueron incluidos la Secretaría General de la ANSEA además de la India, Pakistán, Mongolia y los nuevos miembros de la UE a partir de enero de 2007, Bulgaria y Rumania. Los países mencionados podrán asistir como miembros a la cumbre ASEM 7 a

${ }^{12}$ Gracia Abad Quintanal, "ASEM VI: La renovación del proceso", UNISCI Discussion Papers núm. 13, Madrid, Universidad Complutense de Madrid (2007: 139). Durante la cumbre se adoptó una Declaración sobre el Cambio Climático, ampliando con ello el potencial de temas en la materia que pueden tratarse en el Encuentro. Los 25 países europeos y los 13 asiáticos estuvieron de acuerdo en incorporar la declaración de la Convención de la ONU sobre cambio climático, así como el protocolo de Kyoto para la reducción de partículas de dióxido de carbono. Sin embargo, con excepción del medio ambiente, la toma de posiciones comunes en otros temas relevantes es difícil de identificar (Loewen, 2007: 28-29). 
efectuarse en Pekín en 2008. La presencia de estos países, en especial las potencias nucleares del Sur de Asia (no pertenecientes a ANSEA + 3), dará una mayor complejidad al proceso (Abad Quintanal, 2007: 142-143).

En ASEM puede observarse que tanto la parte europea como la asiática se concentran más en el mantenimiento del vínculo que en la articulación de un diálogo político más o menos efectivo (su flexibilidad es deliberada) o en la centralización de la toma de decisiones. La debilidad institucional del ASEM utiliza al propio aparato de la UE para no manifestarse plenamente (Song, 2007: 76).

El esquema de funcionamiento de ASEM indica que mientras el Consejo Europeo y la Comisión son los representantes de la EU y de la comunidad en el foro interregional, la parte asiática, representada a su vez por dos miembros rotatorios del grupo ANSEA + 3, debe realizar acuerdos intrarregionales previos a su reunión con la parte europea, dando a la región denominada "Asia" un carácter central en el diálogo:

[...] la parte asiática debe, en mayor grado, inventar una "asianidad" sobre la cual construir su posición regional frente a una contraparte ya definida (la UE) y esto deja abierta la cuestión sobre qué tipo de región podría emerger. El interregionalismo puede representar no únicamente la conjunción de dos regiones independientes, sino representar un proceso en el que, a través de la interacción mutua, las regiones de Asia del Este y la UE comiencen a reconocerse mutuamente como tales. Una región puede, de hecho, derivar su propia identidad en parte como resultado de ser aceptada como una "región" por un otro regional discernible y predefinido y existir a través del proceso de esfuerzos recíprocos (Gilson, 2005: 309-310).

Desde la perspectiva constructivista, el interregionalismo puede tener una función cognitiva en tanto define los conceptos de región en medio de la diversidad de los actores.

Sin embargo, la incorporación del ASEM al sistema político de la UE tiene implicaciones más ambiguas. Como veremos, existen dos inter- 
pretaciones sobre este tema: por un lado, se considera que, contando la Unión con una institucionalización mayor que la del grupo ANSEA +3 , los socios asiáticos podrían aprender de sus contraparte europea cómo efectuar una formalización mayor en su propio proceso de construcción regional. Pero, por otra parte, si bien los políticos europeos reconocen la importancia del proceso para las relaciones euroasiáticas (Christopher Dent lo ha resumido en la frase "si no existiera ASEM, debería crearse"), la imagen del ASEM entre los miembros de la Unión y la opinión pública europea es más bien débil.

\section{ASEM y el sistema institucional de la Unión Europea}

Una de las características que parece acompañar a la acción exterior de la UE es la discrepancia entre las percepciones y expectativas que genera su política exterior y las implicaciones reales y legales que la determinan. El contexto en que se desarrolla la acción exterior europea no es algo homogéneo, sino que en su articulación participan diferentes instituciones y actores políticos con distintas competencias entre ellos: existe una UE, una Comunidad Europea, y un conjunto de Estados miembros actuando e interactuando de manera conjunta o individual, o cualquier combinación de estas variables (Govaere, 2006: 97-98). ${ }^{13}$

De la misma forma, cada diálogo interregional tiene una naturaleza distinta como parte de la acción exterior de la UE:

Los diálogos regionales de la UE han cambiado en su formato y estructura en parte por una reflexión de los cambios que han tenido lugar en la integración europea. Por lo general

13 “Es significativo que aún no exista un representante o portavoz en la escena internacional a pesar del artículo 3 del TUE sobre un esquema institucional único al respecto. La Comunidad es representada por la Comisión, mientras que la Presidencia del Consejo representa a la UE. Para conseguir una política exterior coherente y consistente se necesita de la colaboración activa de ambas instancias, o por lo menos una coordinación de sus respectivas actividades externas" (Goveare, 2006: 98). 


\begin{abstract}
"diálogo regional" en la terminología de la UE se refiere a una relación que cubre los elementos tanto económicos como políticos. Desde el punto de vista de las instituciones de la UE esto significa que los mecanismos del primer pilar y del segundo pilar se hallan involucrados (European Background Study, 2006: 142).
\end{abstract}

Diálogos interregionales como el ASEM, el diálogo Euromediterráneo y el establecido con América Latina pertenecen a la política exterior instrumentada tras el Tratado de Maastricht (European Background Study, 2006: 142).

En el caso del ASEM, el mayor reto para la incorporación del diálogo en el sistema institucional de la UE se origina en su carácter híbrido, al situarse entre una estructura pura de relaciones de Estado a Estado y también como parte de las relaciones exteriores comunitarias. Las relaciones de la UE con Asia del Este se orientan por prácticas políticas e institucionales que siguen la misma lógica del sistema de tres pilares de la UE y la incorporación de todos los temas de desarrollo en el pilar económico, mientras que en el pilar político se incorporan los temas de política exterior. Esta división se refleja en el importante papel que desempeña la Comisión Europea en un diálogo informal como ASEM:

La Comisión Europea es otro socio pero no en su función acostumbrada en la acción exterior, es decir como representante de los Estados miembros en los asuntos del primer pilar (a través de la Comunidad Europea), sino en su función de ser un importante actor en las relaciones Asia-Europa en general (European Background Study, 2006: 139).

Ello se debe a que el ASEM no fue creado para dar un seguimiento cotidiano a las relaciones euroasiáticas, sino para articular un diálogo de alto nivel entre los líderes de las dos regiones; lo anterior le ha otorgado al ASEM un carácter un tanto ambiguo como parte de la acción exterior de la UE, condición que se ha fortalecido conforme las relaciones euroasiáticas se han profundizado. 


\section{A) LA COMISIÓN EUROPEA}

De acuerdo con el artículo 3 del Tratado de la Unión Europea, la Comunidad Europea es representada por la Comisión Europea mientras que la Presidencia del Consejo representa a la UE. A su vez, el Tratado de la Unión Europea estipula que la Comisión mantendrá todas la relaciones apropiadas no sólo con ciertas organizaciones internacionales específicas como la ONU y el Consejo de Europa (Govaere, 2006: 102). Las actividades exteriores de la Comunidad frente a los miembros resultan aceptables debido a la función de la Comisión como guardiana de los tratados internacionales establecidos por la Unión, al mismo tiempo que la Comisión trata de legitimar los alcances de la acción exterior de la UE mediante el establecimiento de acuerdos con otras organizaciones internacionales. En el caso del interregionalismo, al apartarse el monopolio de las relaciones de Estado a Estado, "la cooperación grupo a grupo ofreció a la Comisión la oportunidad para desarrollar el proceso de construcción institucional de la CEE/UE" (Forster, 1999: 793). Sin embargo, la Comisión ha encontrado fuertes barreras para trascender las competencias que exigen los miembros estatales de la Unión. Tales barreras han provocado la carencia de una identidad europea común en materia de política exterior y la dificultad de encontrar intereses compartidos, provocando con ello la debilidad institucional de la UE (Richards y Kirkpatrick, 1999: 690). Pero, a pesar de lo anterior, es la propia Comisión Europea la que identifica las características oficiales del ASEM: informalidad (complementando los trabajos realizados en los ámbitos bilateral y multilateral), multidimensionalidad (dando un mismo peso a los tres pilares), asociación entre iguales de sus miembros, y su condición de foro de relaciones de alto nivel. ${ }^{14}$

Desde el punto de vista de la UE, la informalidad y el carácter de Estado a Estado del diálogo en ASEM por una parte, y la profundización de la política exterior común de la UE por otra, parece formar una contradicción en ASEM. Esta contradicción

${ }^{14}$ Véase http:/ / www.aseminfoboard.org/About/ 
afectará las posibilidades para desarrollar a ASEM. La carencia de resultados tanto legales como concretos es frecuentemente mencionada como una de las razones detrás del bajo perfil que el proceso tiene en Europa y del débil involucramiento de los gobiernos europeos en el mismo. Pero, por otra parte, el desarrollo de ASEM en dirección de un foro de negociación formal con instrumentos legales demandaría un cambio en la participación y representación de los miembros entrando en el campo donde se aplican las competencias de la UE (European Background Study, 2006: 145).

\section{B) El CONSEJO EUROPEO}

Tradicionalmente, es la Presidencia de la UE la que se encarga de fijar posturas comunes entre los miembros de la Comunidad y coordinar la agenda que se llevará a cabo con el socio interregional. En el ASEM, la Presidencia rotatoria del Consejo asume un papel como coordinadora del proceso junto a la Comisión Europea; sin embargo, dada la naturaleza híbrida del ASEM, son los Estados miembros de la Unión los que se representan a sí mismos en el foro.

La división de labores entre los dos coordinadores europeos en el ASEM no es similar a otros aspectos de las relaciones entre Asia y Europa. En este caso, el papel de la Comisión no se limita al primer pilar sino que cubre un espectro más amplio de temas en el ASEM. Una estructura de administración intermedia de las relaciones exteriores, ubicada entre la Comisión y el Consejo, como el secretariado del Consejo o el Alto Representante de la PESC, no existe en ASEM (European Background Study, 2006: 141).

\section{C) El Parlamento Europeo}

Al ser el único coordinador permanente en el ASEM, la Comisión Europea ha asumido un fuerte papel coordinador incluyendo áreas en las que comúnmente no podría operar dada la distribución de competencias al interior de la UE. La causa de ello es el carácter político de las resoluciones del ASEM: al no tener un carácter vinculante, dichas resoluciones consensuadas no son tratados susceptibles de ser aprobados 
por el Parlamento (Forster, 1999: 797). Por esa razón la cuestión de Myanmar ofrece consecuencias más visibles en el caso del diálogo EUANSEA, en el que los resolutivos sí deben ser estudiados y aprobados por el Parlamento Europeo (lo que se convierte en el primer obstáculo para el desarrollo de mayores vínculos de cooperación entre ambas organizaciones). Mientras que los acuerdos del diálogo UE-ANSEA deben ser aprobados por el Parlamento Europeo, no hay una obligación así en el caso del ASEM.

Tal situación institucional ha provocado una distorsión de la imagen del ASEM entre la opinión pública europea, pues si bien el Parlamento no posee competencias sustanciales en la PESC o en la formulación de la agenda en política exterior o en la toma de decisiones, sí debe ser informado regularmente por la Presidencia y la Comisión, que le consultan en asuntos fundamentales concernientes a la política exterior (Ambos, 2006: 182).

El hecho que en la mayoría de las ocasiones el diálogo ASEM se lleve a cabo en la política de alto nivel sin duda ha afectado su imagen y visibilidad ante las sociedades europeas. ASEM ha sido criticado por carecer de transparencia parlamentaria en tanto que el Parlamento Europeo y los parlamentos nacionales han sido excluidos de entre los principales participantes (European Background Study, 2006: 151).

\section{Consideraciones finales}

¿En qué medida son relevantes los límites, inconsistencias y tensiones que posee el diálogo político en el ASEM, tomando en cuenta las características y la operación efectiva que demanda la acción exterior de la UE?

Es claro que si nos aproximamos al estudio del ASEM partiendo de la estructura legal de la UE y de la manera en que el ASEM es integrado a su acción exterior aparece una alta tensión política que vuelve inefectivo al Encuentro. El funcionamiento del ASEM contrasta con la intencionalidad de la UE por mantener una alta consistencia en sus procesos institucionales. Pero desde otra perspectiva, se debe considerar que el ASEM, casi desde su origen, fue convirtiéndose en un or- 
ganismo tendiente a discriminar ciertos temas, entre otros los concernientes a la agenda europea de la buena gobernanza global, con tal de no poner en riesgo la naturaleza simétrica del diálogo interregional. $\mathrm{Al}$ menos ésa es una interpretación del énfasis sobre la naturaleza simétrica del diálogo y su marcada orientación por el poder civil de la Unión.

¿En dónde yace el fin práctico para tolerar dichas inconsistencias? Una clave puede residir no solamente en los fines buscados por la UE en establecer un diálogo interregional con Asia del Este, sino en los actores asiáticos que, a fin de cuentas, fueron quienes tomaron la iniciativa para su creación, específicamente en el caso de Singapur y sus motivaciones en ese momento: "Singapur deseaba demostrar que no era solamente un punto comercial sino también un actor diplomático y político en el campo internacional". La iniciativa de Singapur también se conectaba con la creencia de que, aunque no recibía una total aceptación por parte de sus vecinos (Singapur es una ciudad-Estado de mayoría étnica china rodeada por vecinos de etnia malayas, religión musulmana y forma de vida básicamente rural), su prosperidad estaba enlazada con la región y esta presencia era necesaria para promover el comercio y la inversión en la misma (Yeo, 2003: 181). Siguiendo la iniciativa del primer ministro Goh Chok Tong, los trabajos preparativos para la creación del ASEM fueron llevados a cabo a través de la ANSEA. El 19 de marzo de 1995, el Senior Officials Meeting (SOM) de la ANSEA adoptó un informe del gobierno de Singapur que establecía la manera en como se elegirían a los miembros de la cumbre: la ANSEA se encargaría de la designación de los miembros por la parte asiática, mientras que la UE haría lo mismo por parte del lado europeo (Yeo, 2003: 24). Al hablar en nombre de Asia, el gobierno de Singapur podía reafirmar su propio proyecto de construcción nacional en un proceso que puede denominarse "asianización" de la política exterior de Singapur, en la medida en que la noción de "Asia" en el ASEM implica una reinvención de Asia del Este.

Durante los años noventa el regionalismo de Asia del Este recibió un impulso por parte de la idea asianista de que el Sureste y el Noreste de Asia se encontraban unidos por valo- 
res compartidos, originados en tradiciones culturales comunes que los diferenciaba de los occidentales. Esta idea fue una anatema desde la perspectiva de muchos especialistas quienes veían al Sureste y al Noreste de Asia como dos áreas culturalmente distintas (Millar, 2004: 5-6).

Otros autores como Francis Fukuyama criticaron el emergente discurso de los valores asiáticos como un intento de los regímenes autoritarios (o de autoritarismo blando) de la región para evitar una transición política efectiva hacia la democracia y la protección de los derechos humanos.

Líderes asianistas como Mahattir en Malasia y Lee Kuan Yew en Singapur, quienes no sentían una gran atracción por la cultura popular occidental y sus valores democrático-liberales, de hecho intentaron construir sus propias alternativas. Esas alternativas se encontraron en los supuestos valores asiáticos y el respeto a la autoridad, el trabajo duro, la armonía social (diez o quince años antes de que el gobierno chino de Hu Jintao se orientara hacia el discurso de la "armonía"), y la primacía del grupo sobre el individuo, lo cual contrastaba con los percibidos valores occidentales de egoísmo, hedonismo e intemperancia (Miller, $2004: 6$ ).

La llamada Escuela de Singapur de los valores asiáticos tuvo un papel protagónico en impulsar la creación del diálogo euroasiático.

EL ASEM es un ejemplo de cómo en la globalización contemporánea la representación cultural no está determinada por la ideología política, sino que es un instrumento para la organización política, es decir, para la formación de las élites y cuadros políticos (tanto asiáticos como europeos: élites de gobierno en Bruselas y en las metrópolis asiáticas) en el contexto de las condiciones de globalidad y regionalización actuales.

La cultura es introducida en el proceso como un necesario problema para el Encuentro. El requerimiento para "entender" a la cultura recurre, a través de una observación de las 
discusiones en los documentos oficiales de ASEM, bajo otras categorías, particularmente lo político. El entendimiento es problematizado por la diferencia (entre Asia y Europa) y, la diferencia, que resulta ser la base primordial del Encuentro, es definida por la cultura. Igualmente, los términos diferenciados de "Asia" y "Europa" infieren identidades colectivas las cuales preceden al Encuentro, y por ello estructuran las diferencias entre cada uno de los participantes en un orden binario. Europa y no la UE (pensemos que sólo participan los miembros de la UE) es una categoría indicando que lo político se ha convertido en algo codificado e incluso definido en términos de lo cultural (Drake, 2003: 34).

El pilar cultural del ASEM "se ha convertido en un repositorio estructural que es utilizado en un sentido político práctico para organizar la agenda". Lo cultural se convierte en un depósito de temas en los que se problematizan la identidad e intereses de los actores, y temas como la democracia, las sociedades civiles y los derechos humanos van a aparecer bajo la forma del diálogo concerniente a la cultura. Por otro lado, los temas referentes a la soberanía o al funcionamiento de la administración interna de los Estados (como la seguridad o el control de armas) van a pertenecer a la esfera de lo político. ${ }^{15}$

La percepción de la opinión pública europea, al considerarse aleja$\mathrm{da}$, e incluso marginada, del esquema de diplomacia de cumbres del ASEM, es la reacción ante un proceso de formación de élites bajo el esquema político anterior. La formación de una clase política para el diálogo euroasiático tiene como punto de partida la diferenciación de dos proyectos históricos que se encuentran frente a frente en condi-

15 También hay que tomar en cuenta la existencia de una específica cultura de cooperación, la cual se deriva de ciertas predisposiciones y normas culturales compartidas, ya sean imaginadas o concretas. Para Howard Loewen, las diferencias culturales en cuanto a desarrollar el mecanismo de cooperación resultan relevantes: "Los asiáticos tienden a enfatizar el proceso de cooperación y no consideran los resultados como importantes per se. Por ello, los acuerdos no vinculantes y el intergubernamentalismo residen en el corazón de los principios de cooperación de la propia ANSEA. Aquí tenemos una posible explicación a los insuficientes resultados de la cooperación Asia-Europa en tanto que en ASEM persiste un choque entre las culturas de cooperación europeas y asiáticas" (Loewen, 2007:29). 
ciones simétricas, porque es lo más conveniente desde la perspectiva de ambos. Pero el hecho de tolerar inconsistencias, por parte de la UE, forma parte de un proceso histórico más amplio, un proceso que, como menciona Drake:"es donde encontramos la base ontológica de ASEM".

[...] la representación cultural no funciona en este proyecto bajo el viejo sentido de la ideología, para enmascarar o legitimar las verdaderas intenciones de los agentes, ni tampoco es una (ilusoria) expresión de aquellos intereses. En lugar de eso, el proceso ASEM nos da un ejemplo vívido de cómo la organización de la representación cultural produce una estructura de relaciones para completar la tarea referente a la formación y reproducción de élites al proveer un campo en el que las élites políticas puedan operar; un campo de acción más allá de los dictados dados por la soberanía en el sistema internacional y de Estados que funcionan en el proceso económico.

La interdependencia económica euroasiática, determinada por el proceso de regionalización (dictado por los intereses económicos y financieros privados), es el escenario necesario para el proceso político que se desarrolla con cada cumbre de ASEM. De esa forma, el contexto de la globalización otorga la libertad política sobre la cual se desarrolla dicho proceso de formación de élites.

El hecho es que este campo es construido estratégicamente, en lugar de ser una expresión de identidades culturales preexistentes, lo cual es irrelevante. Lo cardinal es que el campo de la representación cultural permite la identificación y el acuerdo sobre el intercambio y la formación de redes necesaria para la formación y reproducción de élites. Ello también permite un proceso de élites políticas que es representado como necesario no en el sentido de expresar estructuras e identidades profundas, sino en un sentido político práctico (Drake, 2003: 37).

La reinvención de "Europa" y de "Asia" a través de una relación simétrica, en la que cada instancia evita convertirse en un objeto del otro, va más allá de las interpretaciones realista o liberal del proceso 
ASEM: mientras que ambas posturas teóricas se aproximan al estudio coyuntural del proceso, una reflexión de tipo histórico/cultural ofrece una explicación sobre los efectos políticos que conllevará a largo plazo la emergencia económica de Asia del Este, un proceso histórico del cual el ASEM ciertamente sólo es un producto coyuntural, en tanto que es una herramienta diplomática de entre varias que la UE se ha preocupado por desarrollar.

La condición poscolonial de los países asiáticos se compone por la representación de un pasado de dominación política, económica y cultural que determinó las relaciones asiático-europeas desde el siglo XVIII hasta mediados del siglo XX. Ese pasado condicionó las representaciones del legado histórico resultante de dicho periodo colonial en la memoria colectiva de aquellas sociedades, en conjunción con los movimientos de liberación a través de los cuales lograron su independencia: una memoria en que las relaciones euroasiáticas siempre fueron desfavorablemente asimétricas. La condición poscolonial es determinante para entender las relaciones entre Occidente y Asia, algo que incluye al régimen dictatorial de los militares de Myanmar y su probable devenir hacia un sistema político inspirado en el autoritarismo blando de algunos de sus vecinos.

Recientemente, el éxito en el crecimiento económico alcanzado por las economías de varios países de la región asiática provocó una aumento en su autoconfianza y legitimó, desde la perspectiva de esos regímenes (algunos de ellos autoritarios), el proyecto de construcción nacional que han elegido, algo que ha conformado un relativismo cultural asiático que rechaza el universalismo de los valores europeos (articulando una crítica orientalista de los valores occidentales desde Asia u occidentalismo).

La hipótesis de los valores asiáticos ha desarrollado su propio proyecto en las relaciones internacionales a través del llamado "Asian way" de integración regional (Hänggi, Roloff y Rüland, 2005: 10). Este "Asian way" se fue conformando con valores como la meritocracia, la flexibilidad, la informalidad, la toma de decisiones consensuadas, la baja institucionalización y el principio de no interferencia en los asuntos internos de los otros países, permitiendo incluso a las élites políticas de la región desarrollar una versión del poder blando 
donde las organizaciones regionales confían en la ley dura (hard law) a la vez que interactúan con otras que suscriben la llamada "ley blanda", el problema se presenta respecto a la variación y la naturaleza de los efectos que distintas culturas de cooperación ejercen sobre los foros de diálogo inter y transregionales, su alcance de acción y su capacidad para resolver problemas (Hänggi, Roloff y Rüland, 2005: 10).

\section{Bibliografía}

Abad Quintanal, Gracia (2007), “ASEM VI: La renovación del proceso", UNISCI Discussion Papers núm. 13, Madrid, Universidad Complutense de Madrid.

Aggarwal, Vinod K. y Min Gyo Koo (2005), "The Evolution of APEC and ASEM: Implications Of New East Asian Bilateralism", European Journal of East Asian Studies, vol. 4, núm. 2.

Alecu de Flers, Nicole y Elfriede Regelsberger (2005), "The EU and Inter-regional Cooperation", en Christopher Hill y Michael Smith (eds.), International Relations and the European Union, Oxford, Oxford University Press.

Ambos, Alicia (2006), "The Institutionalization of CFSP and ESDP”, en Dieter Mahncke et al. (eds.), European Foreign Policy. From Rethoric to Reality, Bruselas, PIE, Peter Lang.

Bobrow, Davis B. (1999), “The US and ASEM: Why the Hegemnon didn't Bark", The Pacific Review, vol. 12, núm. 1.

Chen, Zhimin (2005), "NATO, APEC and ASEM: Triadic Interregionalism and Global Order", Asia Europe Journal, núm. 3.

Comisión Europea (2001), La Gobernanza Europea. Un Libro Blanco, Bruselas, COM.

Dent, Christopher (2004), "The Asia-Europe Meeting and Interregionalism. Toward a Theory of Multilateral Utility", Asian Survey, vol. 44, núm. 2.

Drake, Michael S. (2003), “Representing 'Old Countries': The Strategic Representation of Culture as Heritage in the Asia-Europe Summit Meetings", en Stephanie Lawson (ed.), Europe and the Asia Pacific. 
Culture, Identity and Representations of Region, Londres, Routledge Courzon.

European Background Study (2006), ASEM in its Ten Year Looking Back, Looking Forward. An Evaluation of ASEM in its First Decade and an Exploration to its Future Possibilities, Helsinki, University of Helsinki, Network for European Studies.

Forster, Anthony (1999), "Evaluating the EU-ASEM Relationship: A Negotiated Order Approach", Journal of European Public Policy, vol. 5 , núm. 5.

Gilson, Julie (2005), "New Interregionalism? The EU and East Asia”, Journal of European Integration, vol. 27, núm. 3.

Govaere, Inge (2006), "The External Relations of EU. Legal Aspects" en Dieter Mahncke et al. (eds.), European Foreign Policy. From Rethoric to reality?, Bruselas, PIE, Peter Lang.

Hänggi, Heiner (2000), "Interregionalism: Empirical and Theoretical Perspectives". Conferencia presentada en el taller "Dollars, Democracy and Trade: External Influence on Economic Integration in the Americas", The Pacific Council on International Relations, Los Ángeles, 18 de mayo. Disponible en http:// www.cap.lmu.de/transatlantic/download/Haenggi.PDF

Hänggi, Heiner, Ralf Roloff y Jürgen Rüland (2005), “Interregionalism: A New Phenomenom in International Relations", en Heiner Hänggi (ed.), Interregionalism and International Relations. A Stepping Stone to Global Governance?, Londres, Routledge.

Hettne, Björn y Frederick Soderbaum (2005), "Civilian Power or Soft Imperialism? The EU as a Global Actor and the Role of Interregionalism", European Foreign Affairs Review, vol. 10.

Loewen, Howard (2007), "East Asia and Europe-Partners in Global Politics?", Asia Europe Journal, vol. 5, núm 1.

Miller, John (2004), "The Roots and Implications of East Asian Regionalism", Ocassional Paper, Honolulu, Asia-Pacific Center for Security Studies.

Oudjani, Radhia (2006), "EU-Asia Relations", en Dieter Mahncke et al. (eds.), European Foreign Policy. From Rethoric to reality?, Bruselas, PIE, Peter Lang. 
Reiterer, Michael (2006), "Interregionalism as a New Diplomatic Tool: The EU and East Asia”, European Foreign Affairs Review, vol.11. Richards, Gareth y Colin Kirkpatrick (1999), "Reorienting Interregional Cooperation in the Global Political Economy: Europe's East Asian Policy", Journal of Common Market Studies, vol. 37, núm. 4. Rüland, Jürgen (2006), "Interregionalism and the Crisis of Multilateralism: How to Keep the Asia-Europe Meeting (ASEM) Relevant", European Foreign Affairs Review, vol. 11.

Schünemann, Julia (2006), "Cooperación interregional e interregionalismo: Una aproximación socialconstructivista", Documentos de Política núm. 5, Madrid, Universidad Complutense de Madrid. Disponible en http://www.ucm.es/info/icei/pdf/WP\%200506.pdf

Song, Weiqing (2007), "Regionalisation, Inter-regional Cooperation and Global Governance", Asia Europe Journal, vol. 5, núm. 1.

Yeo, Lay Hwee (2003), Asia and Europe. The Development and Different Dimensions of ASEM, Londres, Routledge. 